\title{
RESULTADOS DA LIMPEZA DE BOCA DO AOD APERAM SOUTH AMERICA COM A LANÇA SLAGLESS CLEAN UP*
}

\section{Resumo}

\author{
Breno Totti Maia \\ Bruno Orlando de Almeida Santos ${ }^{2}$ \\ Fabrício Silveira Garajau ${ }^{3}$ \\ Humberto de Sousa Barcelos ${ }^{4}$ \\ Marcelo de Souza Lima Guerra \\ Maurício Ferreira Coelho \\ Otávio Augusto da Cunha Teixeira
}

$\mathrm{Na}$ conjuntura atual, o foco dos produtores de aço tem se voltado fortemente para a otimização dos recursos e do tempo. O AOD é um convertedor de aços inoxidáveis. A intensa agitação do banho obtida pela lança de topo e injeção de gases por ventaneiras geram projeções ao longo do processo com formação e acúmulo de cascões na parte interna do cone superior. O excesso de cascão prejudica as operações de carregamento e vazamento das corridas. Visando reduzir o acúmulo de cascão, foi desenvolvido para - AOD da Aperam South America, a partir do conceito da lança Slagless um módulo de pós-combustão. Os furos de passagem de oxigênio deste módulo ficam posicionados na região onde se alojam as projeções de metal e escória. Os resultados iniciais obtidos com a Slagless Clean Up desenvolvida mostram uma evolução na qualidade da limpeza da boca do AOD, facilitando a operação de carregamento do forno. Ao longo do período foram feitas alterações na abertura dos furos de pós-combustão para adequação das vazões de sopro. O projeto, agora já na sua fase de conclusão, deverá passar por ajustes na quantidade e na distribuição dos furos do módulo de pós-combustão.

Palavras-chave: AOD; Limpeza de boca; Slagless clean up.

\section{CLEANING MOUTH RESULTS IN APERAM SOUTH AMERICA AOD USING SLAGLESS CLEAN UP}

\begin{abstract}
At the current juncture, focuses of steel producers have focused strongly for the optimization of resources and time. AOD is a converter to stainless steel. Intense bath agitation obtained by top lance and gas injection by tuyeres promote projections throughout process leading to formation and accumulation skulls inside the upper cone. Excess skulls added affects charge and tap. To reduce skull accumulation was developed for AOD Aperam South America, from Slagless cartridge concept a post combustion module. Small oxygen holes are inserted in the upper part of cartridge corresponding to area which happen projections of metal and slag. Initial results obtained with the Slagless Clean Up developed showed an evolution in quality of AOD mouth cleaning, greatly facilitating for loading and tapping operation. Throughout the experimental eriod, some changes were made in opening post combustion to adjust small blow holes. The project, now in its final stages and should undergo adjustments in the quantity and distribution of holes post combustion module.

Keywords: AOD; Mouth cleaning; Slagless clean up.

Eng. Metalurgista, Doutor Pesquisador da Lumar Metals, Sócio da ABM, Belo Horizonte, MG, Brasil.

2 Engenheiro de Materiais, Pesquisador da Lumar Metals, sócio da ABM, Ipatinga, MG, Brasil. (In memoriam).

Engenheiro Mecânico e Pesquisador da Lumar Metals, sócio da ABM, Ipatinga, MG, Brasil.

Engenheiro Metalurgista e Assistente Técnico da Aperam, sócio da ABM, Timóteo, MG, Brasil.

Engenheiro Metalurgista e Analista Consultor da Aperam, sócio da ABM, Timóteo, MG, Brasil.
\end{abstract}




\section{$46^{\circ}$}

\section{INTRODUÇÃO}

Com o objetivo de ampliar a produção e otimizar os custos de produção dos aços inoxidáveis na Aperam Timóteo, o convertedor AOD-L entrou em operação em janeiro de 2002, utilizando o antigo espaço e as principais facilidades existentes no então LD2, que foi desativado naquela época, sendo a produção dos aços elétricos e carbono migrada para o já existente convertedor MRP-L. O projeto do AOD-L contou com várias adequações e substituições nos diversos sistemas, conjuntos e componentes que eram utilizados no LD2. Podem ser destacadas, entre outras, as modificações realizadas nos sistemas de sopro, basculamento, carregamento e exaustão de gases. A Figura 1 apresenta o fluxo atual de produção da aciaria da Aperam [1].

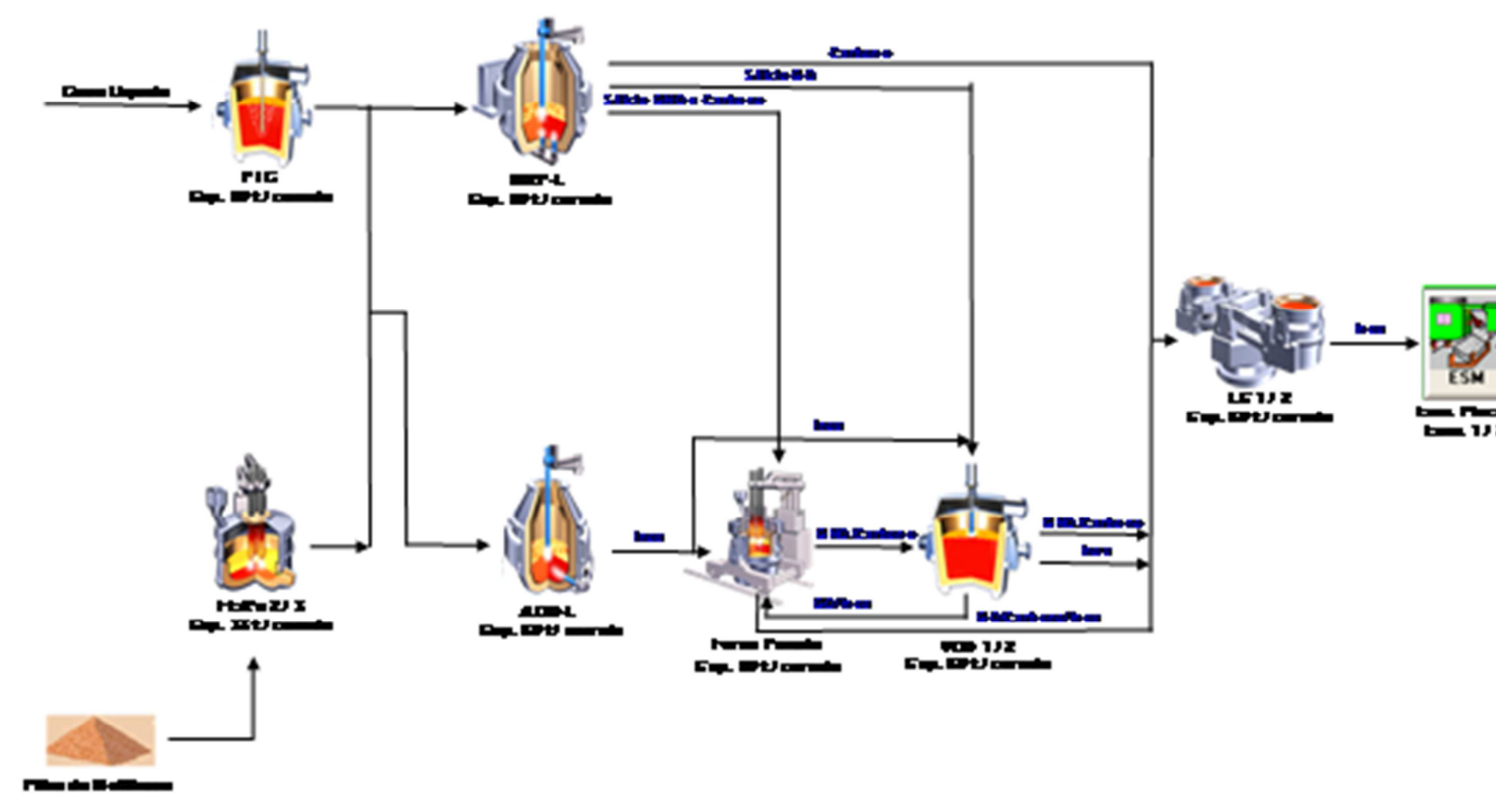

Figura 1 - Fluxo de produção a aciaria da APERAM.

Resumidamente, o processo AOD é uma excelente tecnologia de refino primário e descarburação para fabricação dos aços inoxidáveis. Ele combina o sopro de oxigênio por uma lança de topo com a injeção de misturas de gases via ventaneiras que são inseridas em um dos lados da parte inferior do convertedor, conforme apresentado na Figura 2. 


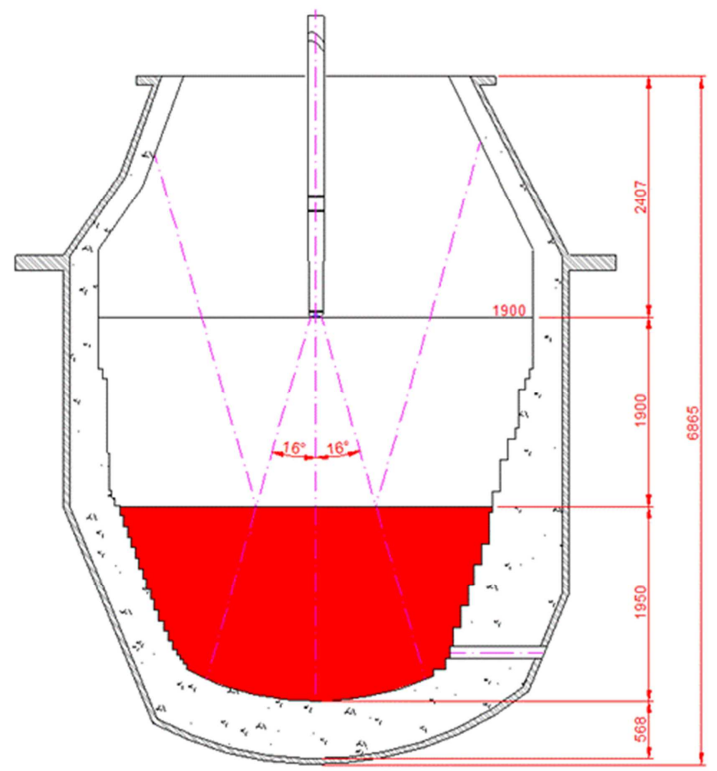

Figura 2 - Corte lateral do convertedor AOD-L.

Esta condição favorece sobremaneira a cinética das reações devido à intensa agitação do banho que pode ser obtida. Durante todo o processo, gases inertes (nitrogênio e/ou argônio) promovem a refrigeração das ventaneiras através da insuflação dos mesmos pela parte externa das ventaneiras que são tubos concêntricos que, na sua parte central, recebem a injeção do gás de processo principal, que é obtido a partir de diferentes misturas de oxigênio com gás inerte, cujas proporções e vazões individuais variam de acordo com cada estágio da fase de descarburação. A partir das fases finais da remoção do carbono, interrompe-se o sopro pela lança de topo, permanecendo apenas a injeção de gases pelas ventaneiras. A etapa derradeira do processo, a redução da escória, visa a recuperação do cromo oxidado durante a descarburação. Neste estágio, apenas gases inertes, especialmente o argônio, são injetados como gases de processo.

\section{MATERIAIS E MÉTODOS}

Visando reduzir o acúmulo de cascão na região da boca do vaso do AOD-L e, assim, manter a área interna da mesma sempre em boas condições para o carregamento, foi desenvolvido, o conceito da lança Slagless Clean Up apresentada na Figura 3.

$\mathrm{O}$ cartucho Slagless [2] consiste em um tubo cônico em cobre de alta de pureza associado a um sistema de interno que permite uma troca térmica efetiva entre a parede de cobre e a água que evita a formação de cascão no corpo da lança de sopro. Acima do tubo de cobre foi um módulo de pós-combustão, denominado Slagless Clean Up. Os furos de passagem de oxigênio deste módulo ficam posicionados, durante o sopro, na região correspondente à área da boca do vaso onde se alojam as projeções de metal e escória conforme apresentado na Figura 4. 


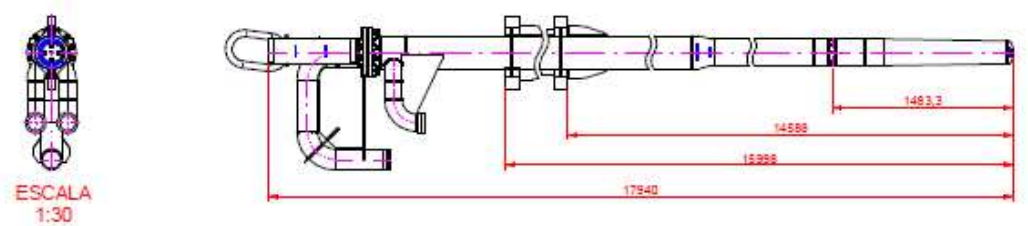

CARTUCHO SLAGLESS CLEAN UP
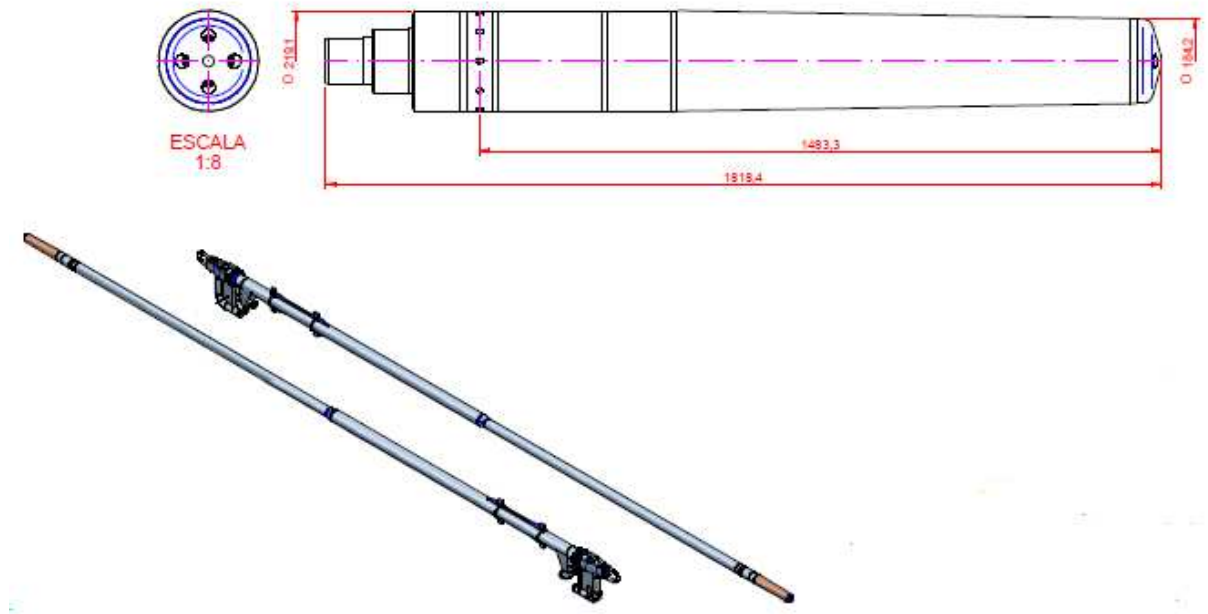

Figura 3 - Cartucho Slagless Clean Up e montagem na lança de sopro.

Módulo de póscombustão com 6 furos

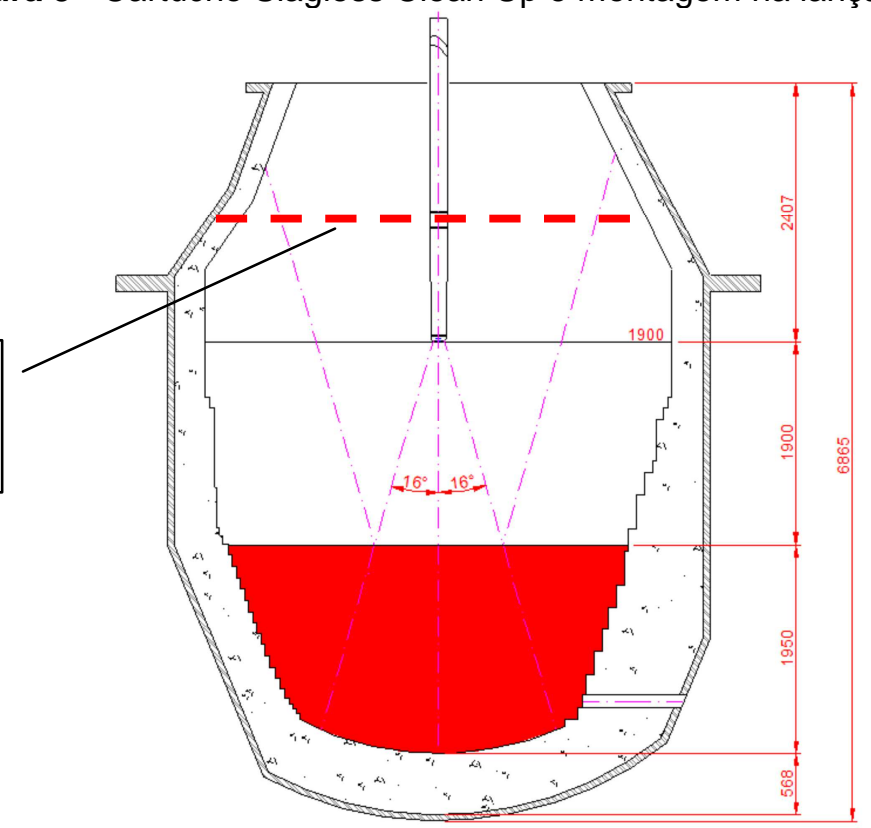

Figura 4 - Convertedor a AOD e posicionamento furos pós-combustão Slagless.

A montagem realizada no carro à esquerda dos mangotes com objetivo de orientar a o posicionamento lança Slagless Clean Up respeitando a distribuição de furos em relação ao carregamento do AOD conforme mostrado através da vista superior esquematizado na Figura 5. 


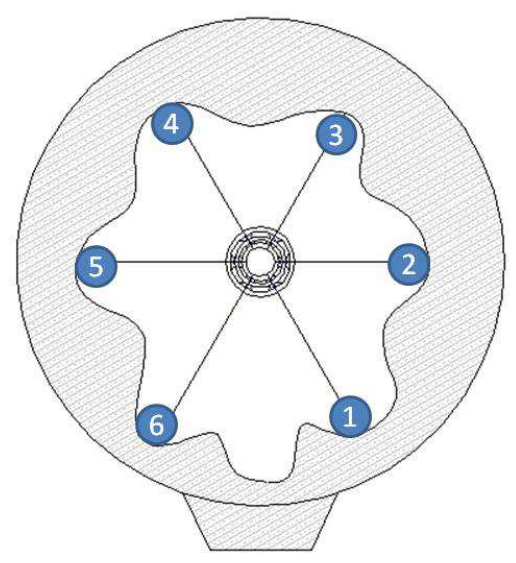

Figura 5 - Distribuição dos furos de pós-combustão ao redor do boca do AOD-L.

\section{RESULTADOS E DISCUSSÃO}

O cartucho Slagless Clean Up teve um bom desempenho de corridas, sendo retirado preventivamente de uso, porém sendo mantida como opção reserva. A Tabela 1 apresenta a evolução da vida do cartucho com o passar das campanhas do AOD-L.

Tabela 1- Histórico das campanhas da experiência inicial com a utilização da lança Slagless Clean Up no AOD-L.

\begin{tabular}{|c|c|c|c|c|}
\hline $\mathrm{N}^{\circ}$ Seq. & $\begin{array}{c}\mathrm{N}^{\circ} . \\
\text { Campanha } \\
\text { AOD-L }\end{array}$ & Período & $\begin{array}{c}\mathrm{N}^{\circ} \text {. Corridas } \\
\text { Slalgess } \\
\text { Clean Up }\end{array}$ & $\begin{array}{c}\text { Vida } \\
\text { Acumulada }\end{array}$ \\
\hline 1 & 436 & $\mathrm{nov} / 13$ & 55 & 55 \\
\hline 2 & 437 & $\mathrm{nov} / 13$ & 16 & 71 \\
\hline 3 & 451 & $\mathrm{abr} / 14$ & 76 & 147 \\
\hline 4 & 455 & $\mathrm{mai} / 14$ & 92 & 239 \\
\hline 5 & 456 & $\mathrm{mai} / 14$ & 94 & 333 \\
\hline 6 & 457 & $\mathrm{mai} / 14$ & 59 & 392 \\
\hline 7 & 458 & $\mathrm{jun} / 14$ & 71 & 463 \\
\hline 8 & 459 & $\mathrm{jun} / 14$ & 75 & 538 \\
\hline 9 & 460 & $\mathrm{jun} / 14$ & 74 & 612 \\
\hline 10 & 462 & $\mathrm{jul} / 14$ & 77 & 689 \\
\hline 11 & 463 & $\mathrm{jul} / 14$ & 81 & 770 \\
\hline 12 & 464 & $\mathrm{jul} / 14$ & 64 & 834 \\
\hline 13 & 465 & $\mathrm{ago} / 14$ & 54 & 888 \\
\hline 14 & 466 & $\mathrm{ago} / 14$ & 10 & 898 \\
\hline 15 & 467 & $\mathrm{ago}-\mathrm{set} / 14$ & 83 & 981 \\
\hline
\end{tabular}

\section{1 - Perfil de Cascão antes da Slagless Clean Up}

Campanha 461 soprada com lança convencional (Lança 5), foi utilizada para avaliação e comparação da evolução do cascão na boca com lança sem módulo Slagless Clean Up conforme apresentado na Figura 6. 


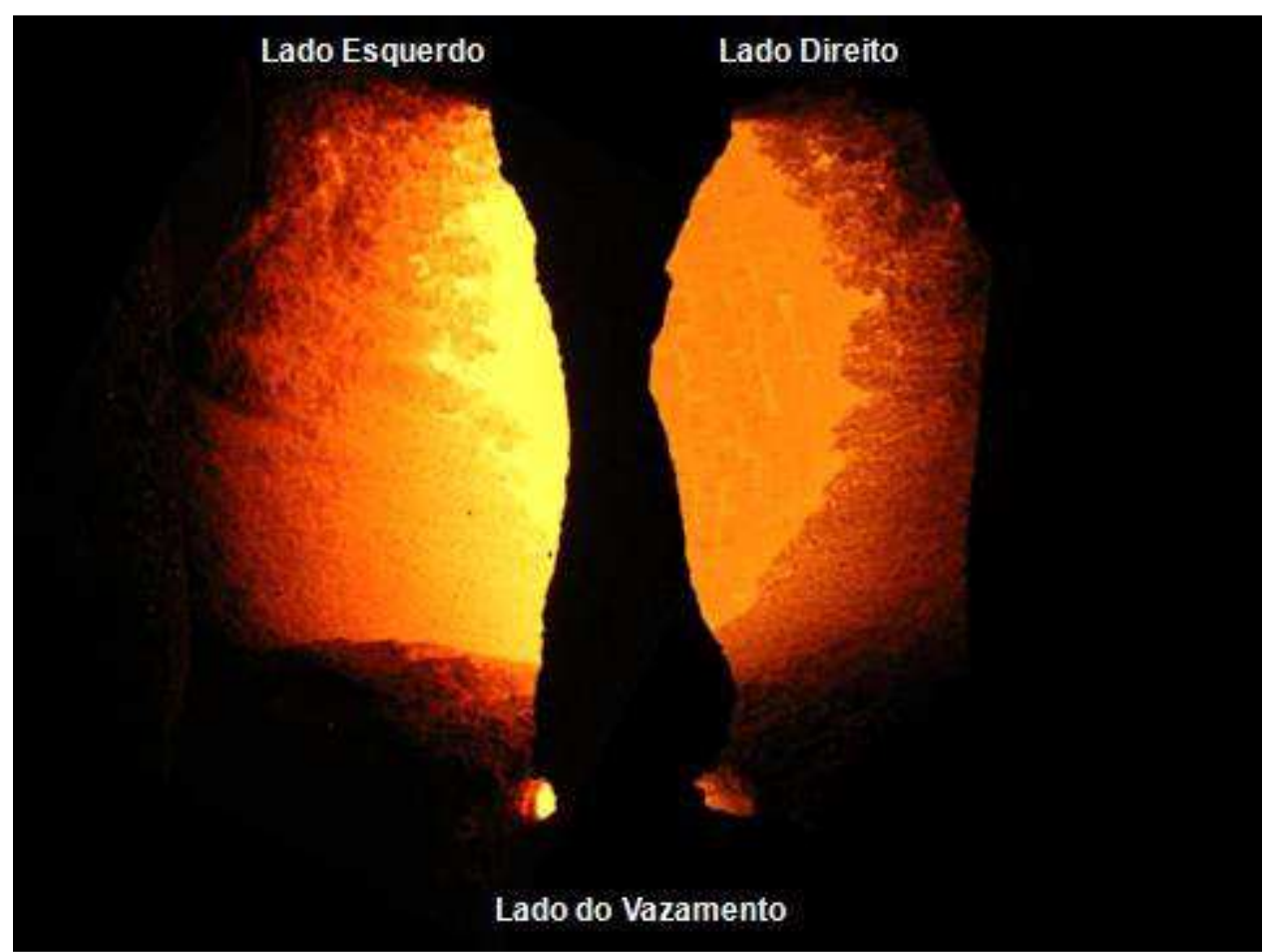

Figura 6 - Formação de cascão de boca AOD-L com lança tradicional.

\section{2 - Campanhas Slagless Clean Up}

Devido o aumento na eficiência da troca térmica um dos principais parâmetros observados no início da operação de um cartucho é perfil do delta de temperatura, conforme apresentado na Figura 7.

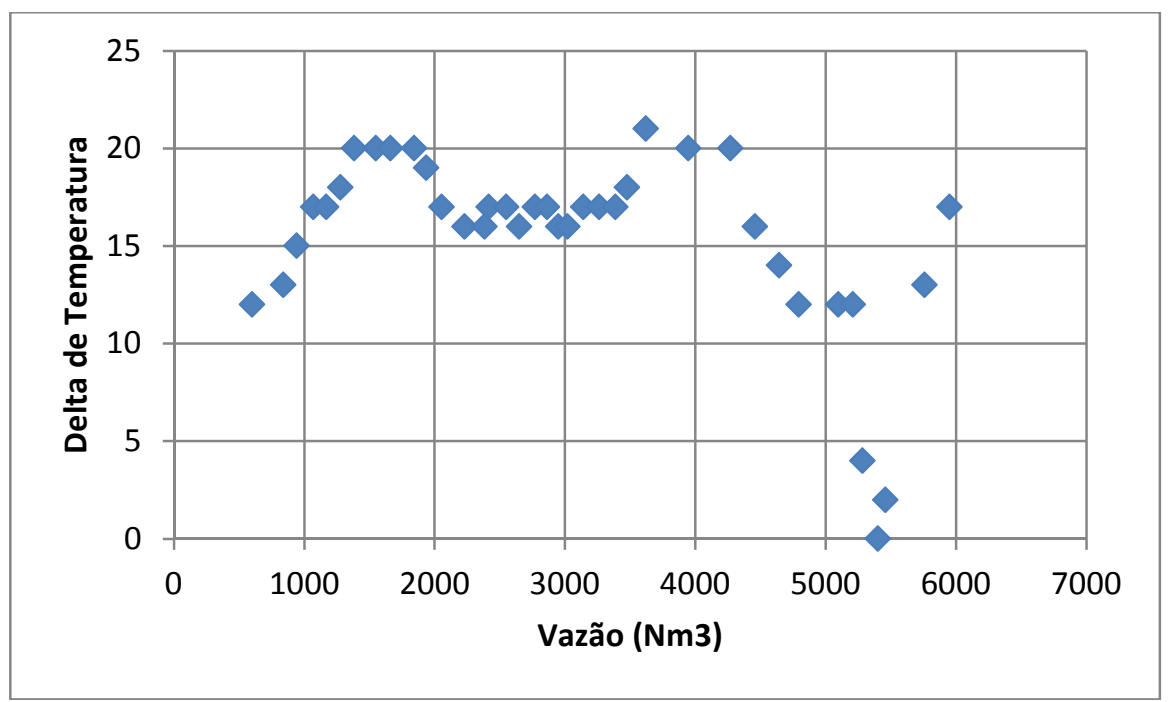

Figura 7 - Perfil da temperatura da água ao longo da corrida.

Foi realizado ajuste permitindo o máximo de delta de temperatura em $28^{\circ} \mathrm{C}$. Os momentos de redução de temperatura apresentados no gráfico está correlacionado com o momento de queda do material dos silos aéreos que ocorre apenas do lado do MRP-L, ou seja, a direita do AOD. Vazão de água inicial foi de $108 \mathrm{Nm}^{3} / \mathrm{h}$ a $9 \mathrm{bar}$ de pressão estabilizando em $101 \mathrm{Nm}^{3} / \mathrm{h}$ após 50 corridas com a lança. Vazão oxigênio foi estabelecida em $120 \mathrm{Nm}^{3} / \mathrm{min}$. em função da qualidade $30 X X$, sendo a 
vazão máxima $150 \mathrm{Nm}^{3} / \mathrm{min}$. Após 55 corridas foi realizada uma análise do perfil de desgaste sendo conforme desgaste conforme Figura 8.

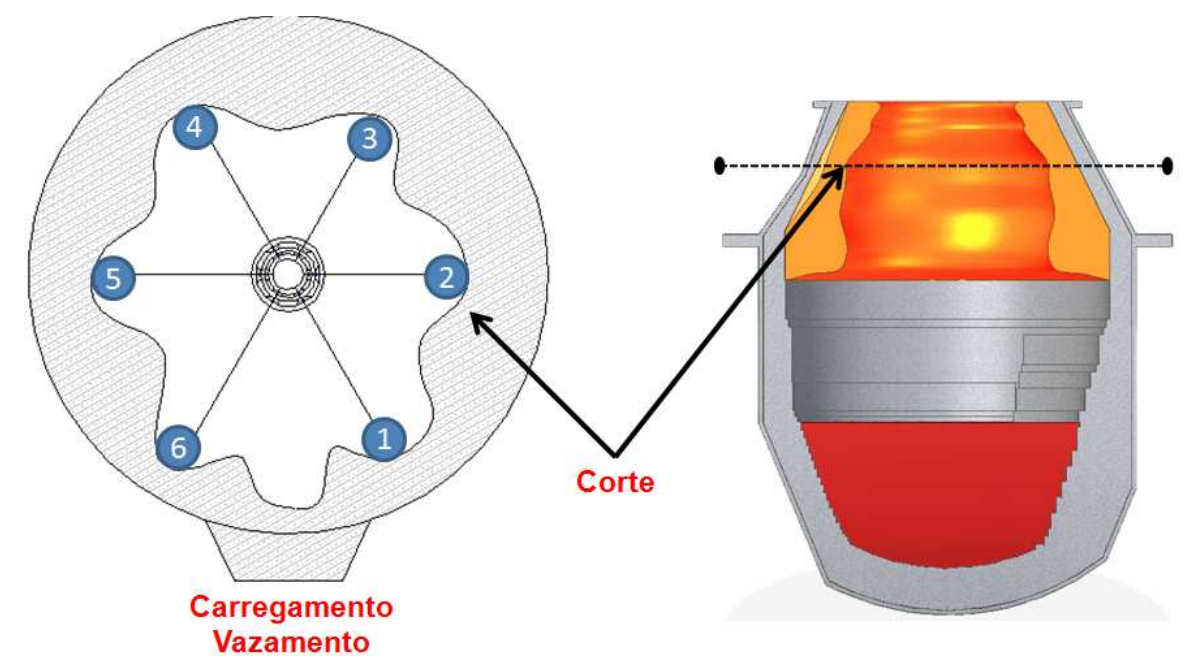

Figura 8 - Perfil de limpeza da Slagless Clean Up início da campanha e aspecto da formação de cascão após.

Fato foi observado na posição da calha (chute) de adição de materiais. Além de reduzir a temperatura da água de circulação da lança, os materiais adicionados também comprometeram o desempenho dos bicos de pós-combustão 1 e 2 provocando o aumento do cascão na região dos munhões do lado direito. Os materiais adicionados são extremamente pesados e duros e provocaram amassamentos tanto no módulo de pós combustão quanto no tubo de aço. A partir dessa constatação foi proposto opções de aumento do diâmetro de saída para promover uma maior vazão nas saídas 1 e 2 conforme Tabela 2.

Tabela 2- Opção de diâmetro de furo de saída x vazão.

\begin{tabular}{|cc|}
\hline Diâmetro Externo (mm) & Vazão $\left(\mathbf{N m}^{\mathbf{3}} \mathbf{~} \mathbf{h}\right)$ \\
\hline 4,2 & $48 /$ Furo \\
\hline 5 & $65 /$ Furo \\
\hline 5,5 & $79 /$ Furo \\
\hline
\end{tabular}

Realizado o ajuste da posição de montagem e alteração dos furos da lança Slagless Clean Up, apresentado comparativamente na Tabela 3, sendo alterado também a posição 4 preventivamente.

Tabela 3 - Comparativo de dimensional antes e depois do ajuste da Slagless Clean Up.

\begin{tabular}{|cc|c|}
\hline & \multicolumn{2}{c|}{ Diâmetro Externo (mm) } \\
\hline Posição & Incial & Final \\
\hline 4 & 4,2 & 5,0 \\
\hline 5 & 5,0 & 5,5 \\
\hline 6 & 5,0 & 5,5 \\
\hline
\end{tabular}

\section{3 - Perfil de Cascão após Slagless Clean Up}

A lança Slagless Clean Up operou em função das necessidades da APERAM e em determinados momentos eram feitos registros de acompanhamento do desempenho. Com 499 corridas, entrando em operação após a 15a corrida da campanha 458 do 
AOD com 47 corridas era possível obervar cavidades formadas pelos furos do módulo de pós-combustão, apresentado na Figura 9.

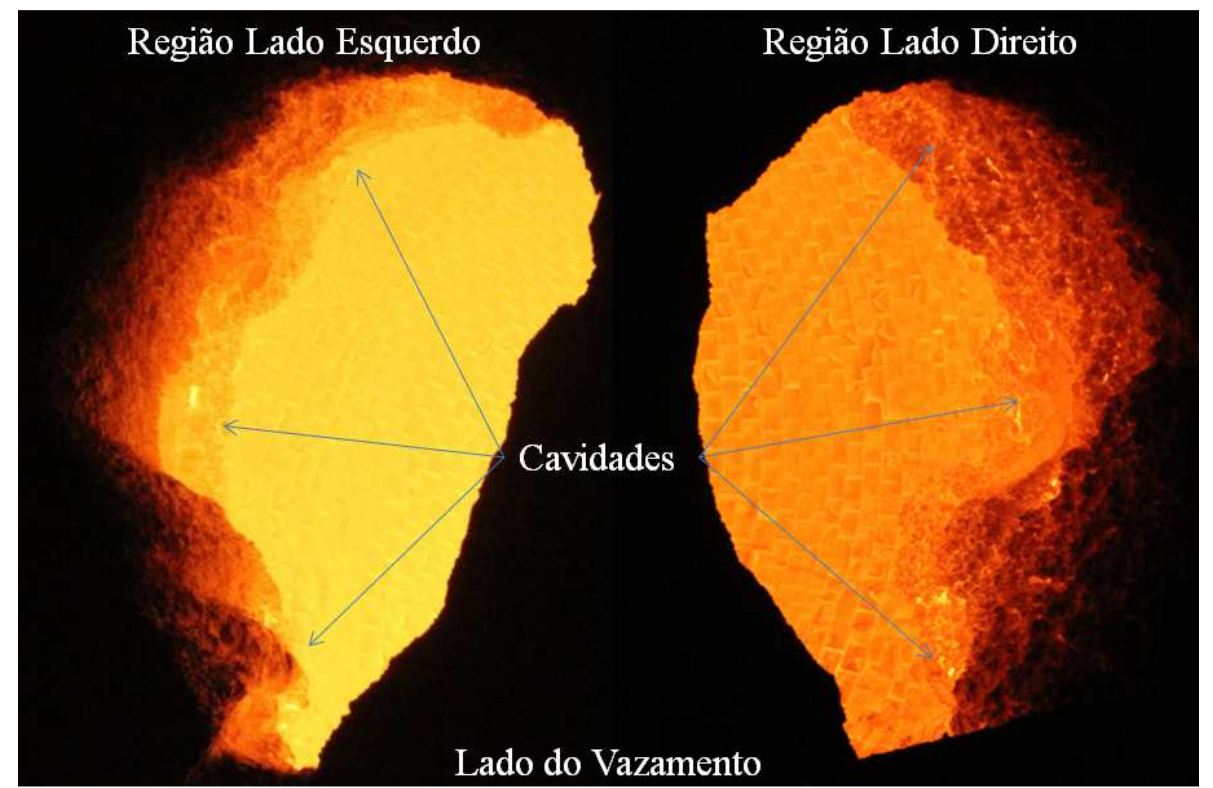

Figura 9 - Foto da formação de cavidades da pós-combustão. AOD com 47 corridas na campanha 458. Slagless Clean Up com 499 corridas.

Lança Slagless CleanUp com 542 corridas, entrando em operação após na 15 corrida da campanha 460. O AOD com 87 corridas na campanha apresenta cavidades formadas pelos furos do módulo de pós-combustão, mostrado na Figura 10.

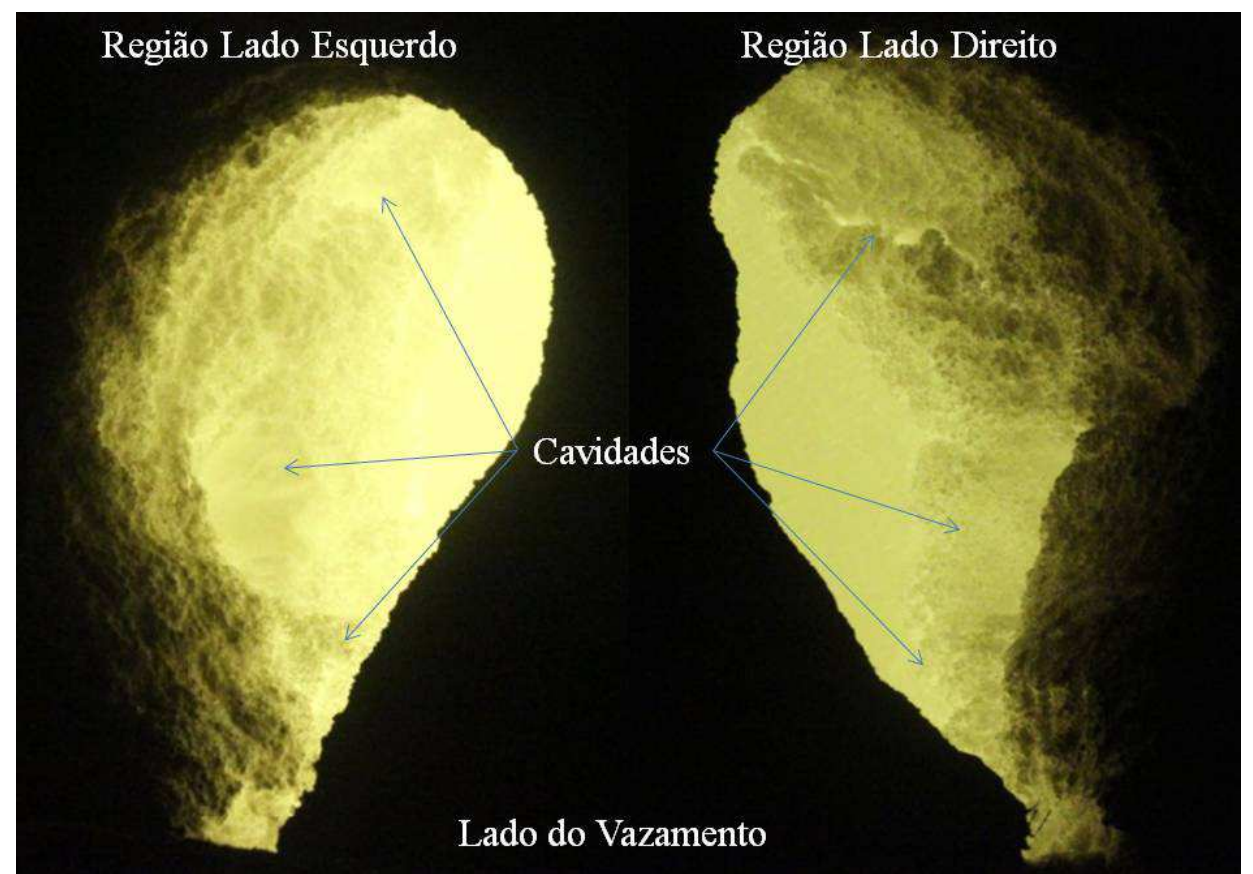

Figura 10 - Foto da formação de cavidades da pós-combustão. AOD com 87 corridas na campanha 460. Slagless Clean Up com 542 corridas.

\section{4 - Comparação do efeito da Slagless Clean Up na Limpeza de Boca do AOD}

O efeito da Slagless CleanUp em comparação com a lança tradicional, pode ser visto na Lado direito do AOD-L ou MRP. 


\section{$46^{\circ}$}

Figura 11. Na Lado direito do AOD-L ou MRP.

Figura 11, o lado esquerdo das imagens está uma imagem da formação de boca com lança tradicional e na direita com o uso da Slagless Clean Up.

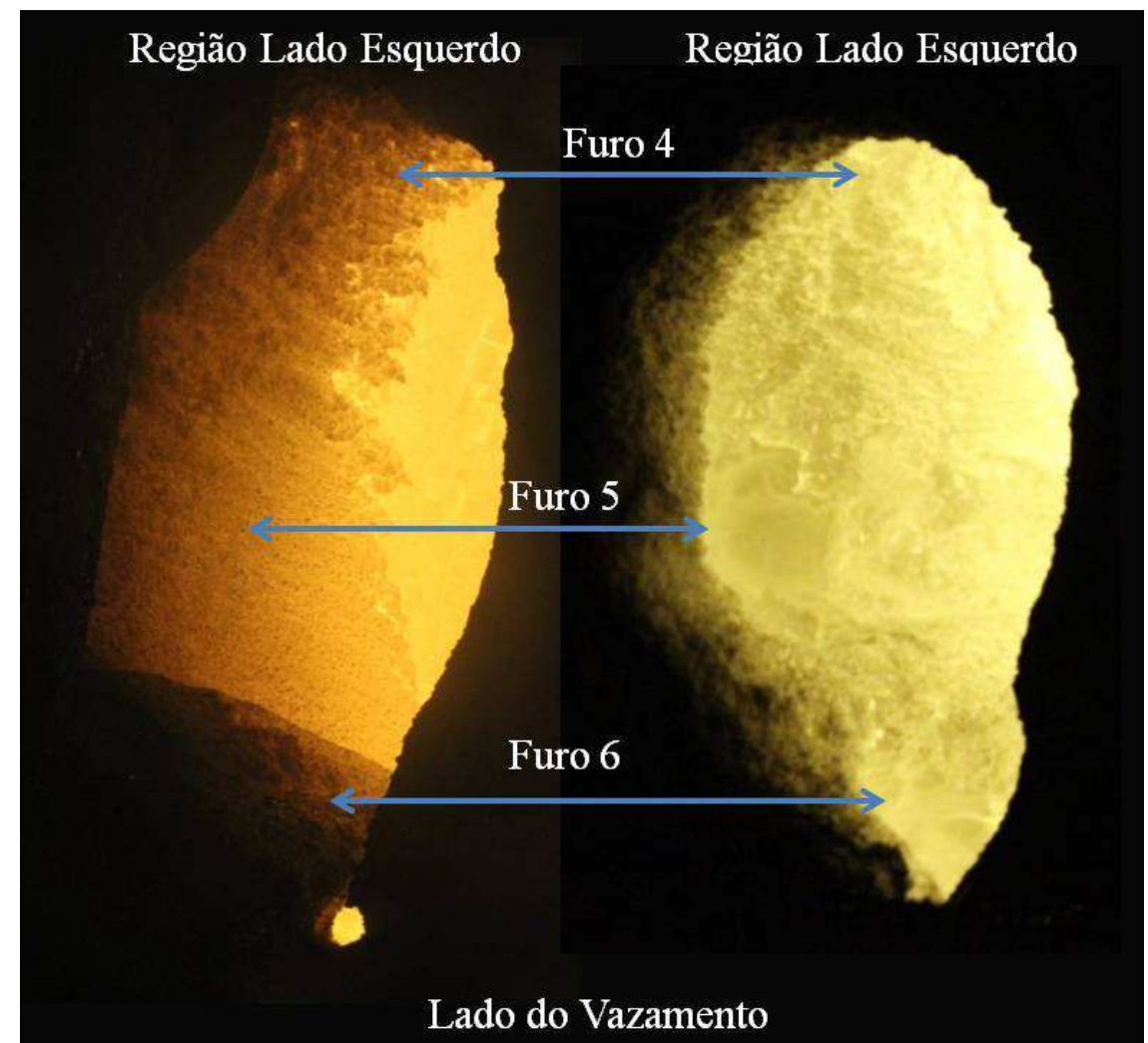

a) Lado esquerdo do AOD-L ou lado do Pré-Tratamento de Gusa (PTG).

Região Lado Direito

Região Lado Direito

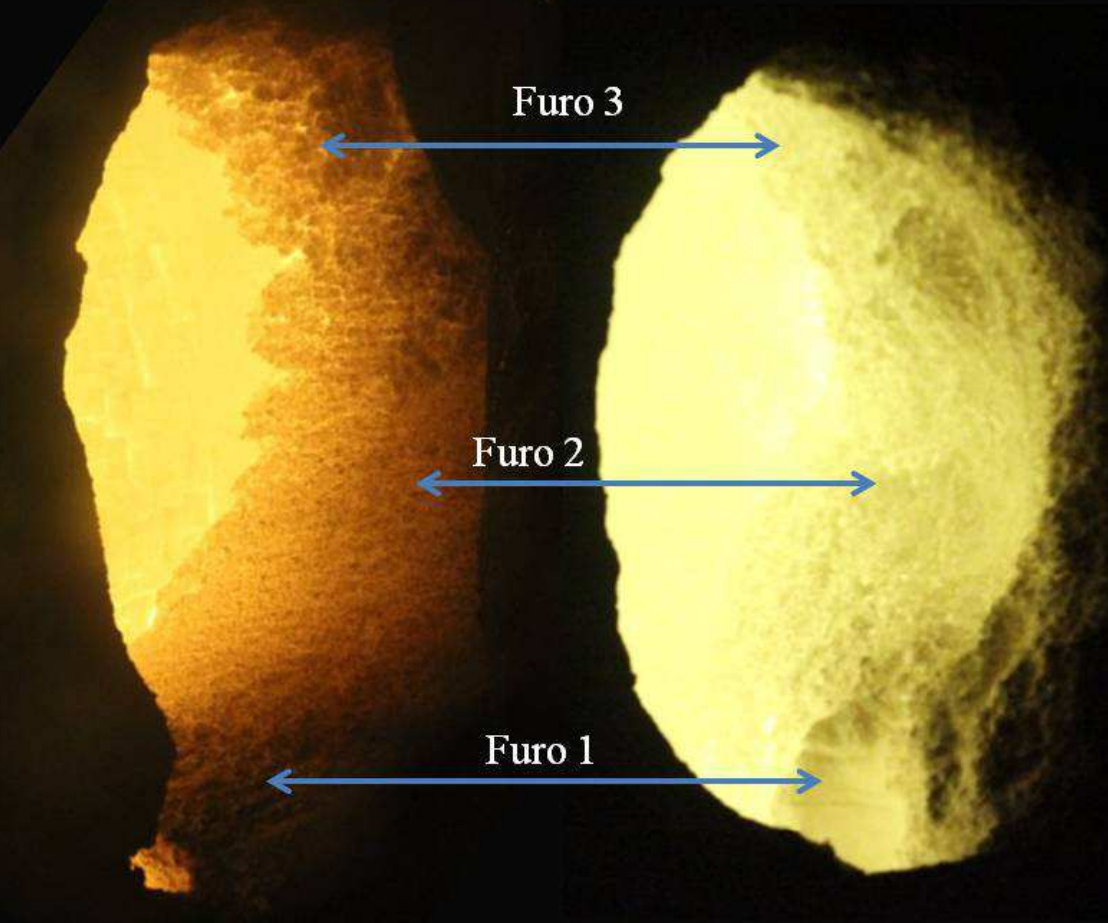

Lado do Vazamento

b) Lado direito do AOD-L ou MRP. 
Figura 11 - Foto do efeito da Slagless CleanUp em comparação com a lança tradicional. a) Lado esquedo (PTG) e b) Lado direito (MRP).

Na Figura 11, é possível notar o efeito de limpeza, evitando o acumulo de material na boca do AOD-L através das cavidades formadas no material aderido. Esse controle e manutenção das condições da boca significa redução no tempo de troca do AOD-L uma vez que o peso final é inferior à capacidade da ponte rolante, permitindo rapidez na troca. Fato comprovado por ocorrências nas campanhas $464 \mathrm{e}$ 465 do AOD-L quando foi necessário a retirada fora de programação e o peso foi inferior a 190t, capacidade limite para retirada do AOD-L sem a necessidade de limpeza prévia da boca.

\section{5 - Integridade da Slagless Clean Up}

Com 611 corridas, foi inspecionada a Lança Slagless CleanUp, conforme Figura 12. As características físicas estão preservadas, onde foi avaliado a deformação de cada furo e constatado menor deformação nos furos 2 e 3.
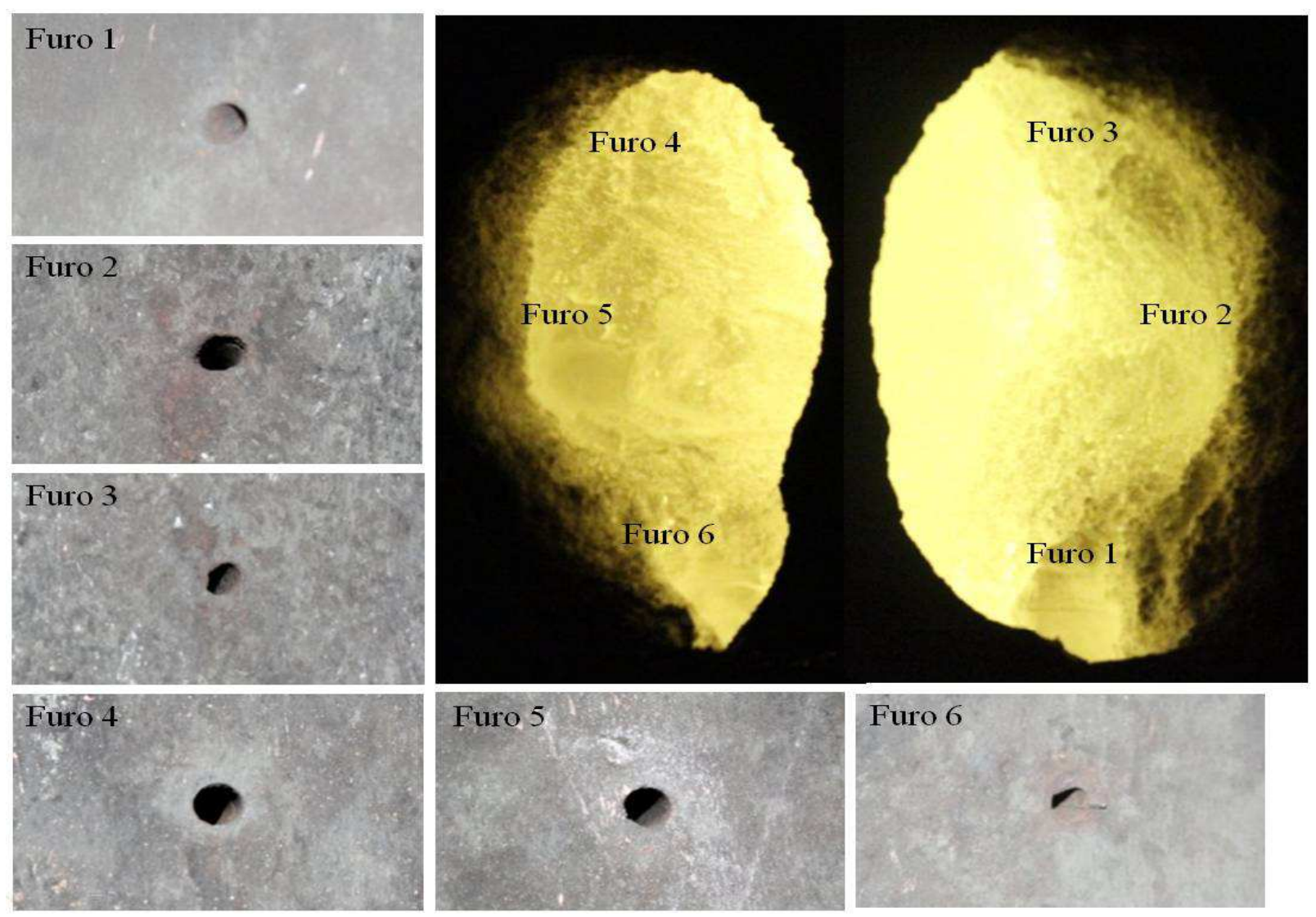

Figura 12 - Foto de inspeção de integridade dos furos de pós-combustão e cavidades formadas no AOD-L. Slagless Clean Up com 611 corridas.

Também foi avaliada a condição dos bicos de sopro da Slagess conforme apresentado na Figura 13. 


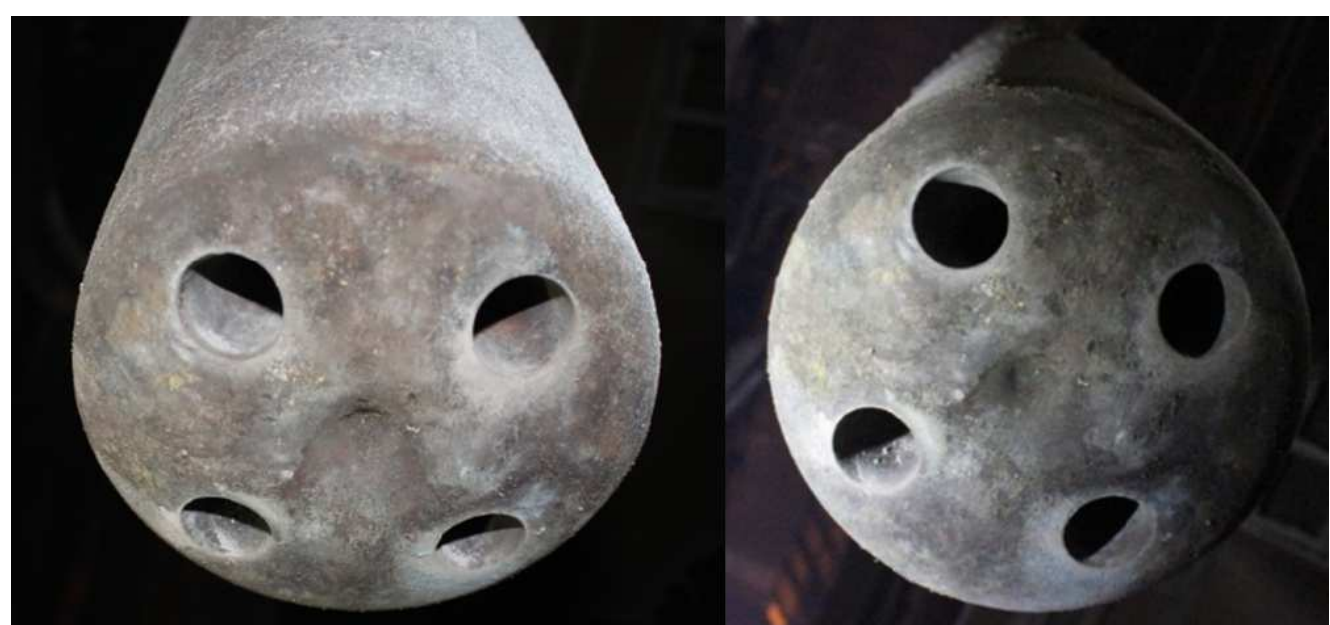

Figura 13 - Foto do aspecto dos bocais principais após 611 corridas na Slagless Clean Up.

Mesmo com um número elevado de corridas quando comparado com bico tradicional, em torno de 350 corridas, o bocais apresentam ótimo estado de conservação, notadamente pela preservação das dimensões de saída de cada bocal e face do bico.

No período de teste da Slagless Clean Up não foi realizado um levantamento do número de cascões de lança devido ao número inexpressivo de ocorrências, apresentado de forma comparativa na Figura 14.
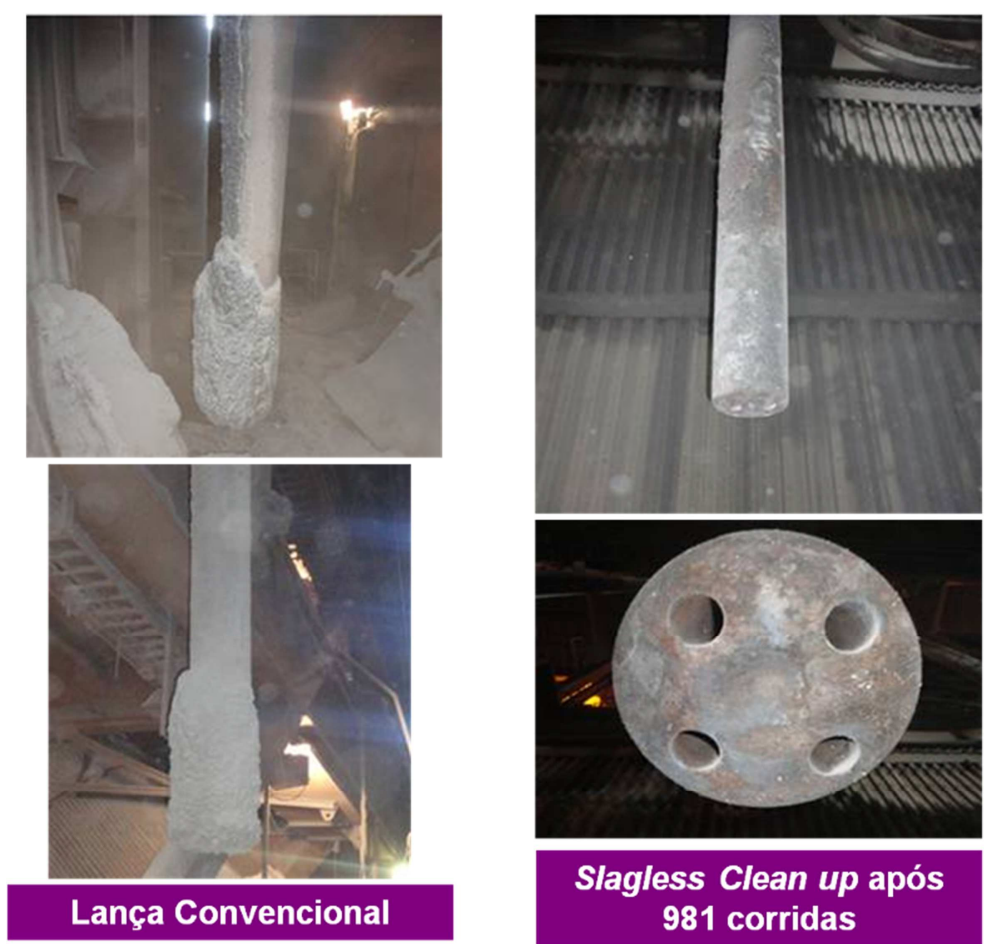

Figura 14 - Comparação de formação de cascão entre lança convencional e Slagless Clean Up.

Na Figura 14 é possível observar tanto o comportamento característico de formação de cascão da lança convencional quanto à preservação do cartucho após 981 corridas. 


\section{CONCLUSÃO}

As principais conclusões são:
a) A Slagless Clean Up atingiu o objetivo de reduzir o cascão de boca do AOD- $\mathrm{L}$;
b) A redução do cascão do AOD-L teve influência na redução do peso do vaso, permitindo reduções nos tempo de troca;
c) A Slagless Clean Up permite ajustes de vazões nos furos de pós-combustão conforme a necessidade de limpeza da boca dos reatores a oxigênio;
d) $O$ cartucho com vida aproximadamente 3 vezes superior quando em comparação com a lança tradicional;
e) Bocais principais e furos de pós-combustão com integridade dimensional após 981 corridas;
f) Slagless Clean Up não apresentou formação de cascões na lança;

\section{PRÓXIMOS PASSSOS}

Avaliar a possibilidade de aumentar o número de furos para uniformizar o ataque ao cascão de boca do AOD-L, conforme proposta da Figura 15.
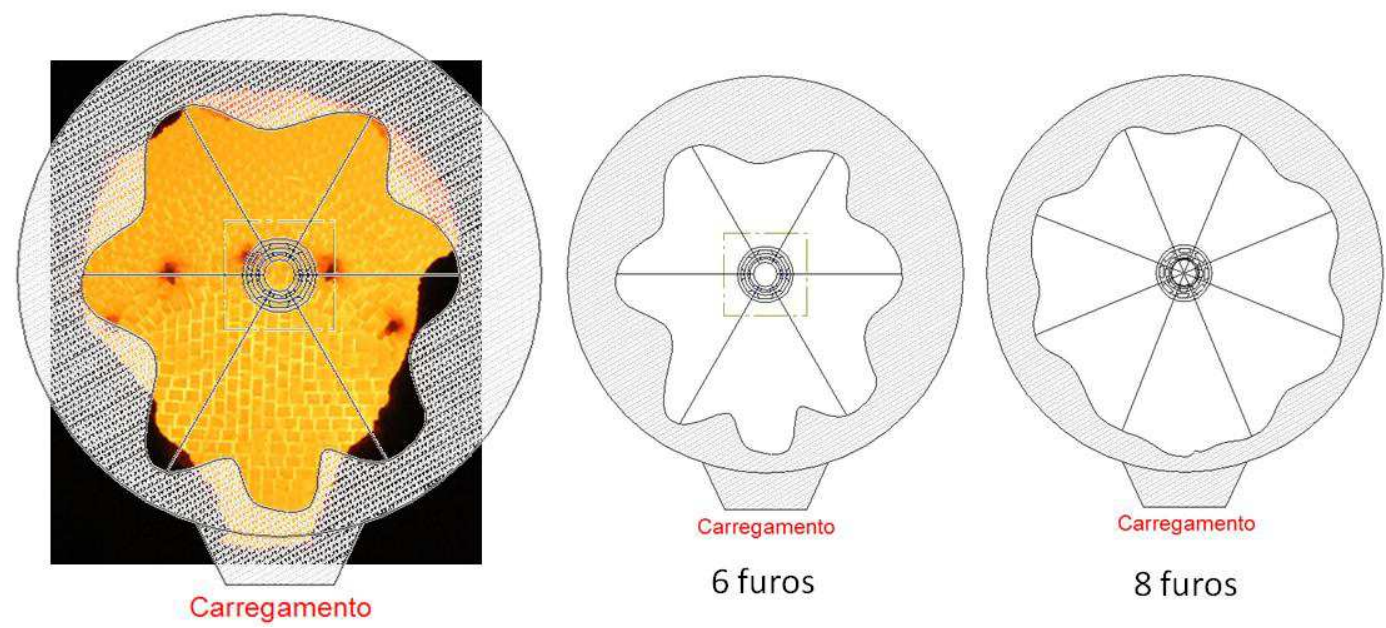

Figura 15 - Proposta de aumento de 6 para 8 furos de pós-combustão.

\section{Agradecimentos}

Os autores a Lumar Metals pelo incentivo a pesquisa. Ao Bruno Alves pelo empenho e qualidade das fotos.

\section{REFERÊNCIAS}

1 Filho, D. S. F., Marin, H., Silva, J. M. F., Porto, R., Análise sobre a Redução do Pick-Up de Nitrogênio na Produção de Aços Inoxidáveis. Anais do $42^{\circ}$ Seminário de Aciaria Internacional, Salvador, Bahia, Maio 2011.

2 Sardinha, I. A., Slagless Lance Recent Results at BOF Steel Plants Have Shown a Better Flexibility in Oygen Blowing. Anais do $6^{\text {th }}$ European Oxygen Steelmaking Conference, Stockholm, Sweden, 2011. 